\title{
The thermal response of heat storage system with paraffin and paraffin/expanded graphite composite for hot water supply
}

\author{
P. Zhang*, L. Xia, R.Z. Wang \\ Institute of Refrigeration and Cryogenics, Shanghai Jiao Tong University, Shanghai 200240, China \\ * Corresponding author. Tel.: +86-21-34205505; fax: +86-21-34206814 E-mail: zhangp@sjtu.edu.cn (P. \\ Zhang)
}

\begin{abstract}
The low thermal conductivity of phase change material (PCM) leads to low heat storage/retrieval rates. The expanded graphite (EG) was used to enhance the thermal conductivity. EG/paraffin composite with the $7 \%$ mass fraction of EG was prepared as a good candidate for the latent thermal energy storage (LTES) system. A shell and tube LTES system built for room heating and hot water supply in a family was experimentally investigated. The paraffin and paraffin/EG composite were used as the heat storage material, respectively. The experimental results indicated: The utilization of EG/paraffin composite PCM greatly improved the heat storage/retrieval rates of the LTES system. The LTES system with paraffin/EG composite showed a $44 \%$ reduction in heat storage duration and a nearly $69 \%$ reduction in the retrieval duration, respectively, compared to those for the system using pure paraffin. The most outstanding advantage, for the LTES system filled with paraffin/EG composite, was that the outlet temperature of water can be maintained at a higher level for a longer term than that with paraffin. However, the LTES system filled with EG/paraffin composite did not show an obvious advantage in the step-by-step heat retrieval mode, compared with paraffin.
\end{abstract}

Keywords: Latent thermal energy storage, Paraffin/expanded graphite composite, Heat storage/retrieval rate

\section{Introduction}

In a latent thermal energy storage (LTES) system by solid-liquid phase change, energy is stored during melting while it is retrieved during solidification of a phase change material (PCM), thus a LTES system with a good performance requires that the PCM possesses the appropriate phase change temperature, high heat storage density and high thermal conductivity. Besides, a good LTES system also lies on a rational structure design of the system which will decide the filling capacity of PCM and the heat exchange surface.

Based on an extensive study by Lane et al. [1] there are about 20,000 substances with the melting point in the range 10-90 oC. Majority of them was abandoned for application due to improper melting point, melting with decomposition or lack of essential reference data [2]. Among these PCMs, normal paraffin of type $\mathrm{C}_{n} \mathrm{H}_{2 n+2}$ has shown outstanding performance for application in LTES systems for solar heating and cooling [3-4]. This is because of its appropriate melting point, large latent heat, low cost, high stability and compatibility, and a low negative environmental impact. Despite the many desirable properties of paraffin, its low thermal conductivity, generally below $0.4 \mathrm{~W} /(\mathrm{m} \cdot \mathrm{K})$, is one of the major drawback.

The PCM containers with different geometries have their own advantages and disadvantages. Various LTES techniques have been developed and various encapsulations have been used in LTES systems. Two geometries commonly employed as PCM containers are the rectangular and cylindrical containers [5]. In particular, cylindrical containers accounts for more than $70 \%$ in all the used LTES system which commonly involves the three modes. The first is the heat storage unit in which the PCM fills the shell and the heat transfer fluid (HTF) flows through the central tube [6-8]. In the second mode, the PCM fills the tube and the HTF flows parallel to the tube [9]. The third cylinder mode is the shell and tube system [10,11]. 
In the present work, expanded graphite (EG), with high thermal conductivity, was added into PCM to form a kind of composite phase change material and to enhance the heat transfer of the inner PCM. EG/paraffin composite PCM with 7\% mass fraction of EG was prepared. This ratio was considered as the balance by compromising the heat transfer enhancement and latent heat storage capacity [12]. The EG/paraffin composite PCM was filled in the stainless steel tubes, and then these LTES tubes were compactly arranged in a tank. As a comparison, the paraffin was also used in this system as the heat storage material. The heat storage and retrieval performance of this LTES system, filled with EG/paraffin composite and paraffin, were experimentally tested, respectively. The influence of the HTF flow rate on the performance of the LTES system was also investigated. Moreover, two heat retrieval modes viz.: continuous and step-by-step heat retrieval, which were commonly used in the utilization of LTES system, were executed respectively for testing the heat retrieval performance of the LTES system with two PCMs.

\section{Experimental setup and procedure}

The PCM used in this study was technical grade paraffin with the purity of $99 \%$ and a melting temperature of $62{ }^{\circ} \mathrm{C}$. The EG was prepared by making the raw expandable graphite (mesh 80, type KP80, from Qingdao Tianhe Graphite Co. Ltd, China) subjected to heat treatment in a furnace at $700{ }^{\circ} \mathrm{C}$ for a duration of 15 minutes. These paraffin and EG were used to prepare EG/paraffin composite. The paraffin was heated to a temperature of $85{ }^{\circ} \mathrm{C}$, in order to be liquefied, after which the liquid paraffin was impregnated into EG and was stirred using a roll mixer. Then, the EG/paraffin composite with 7\% mass fraction of EG was obtained.

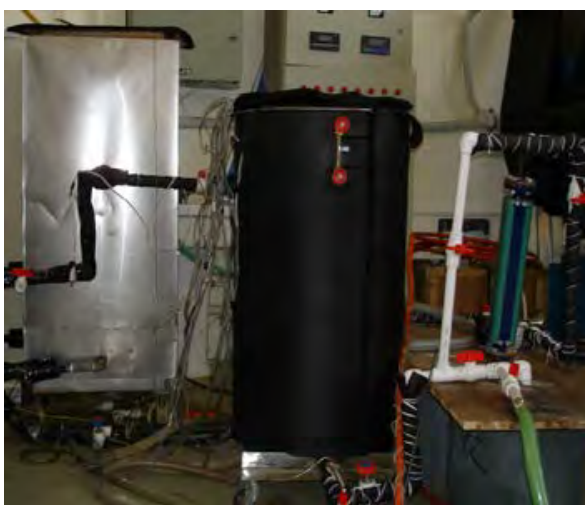

(a)

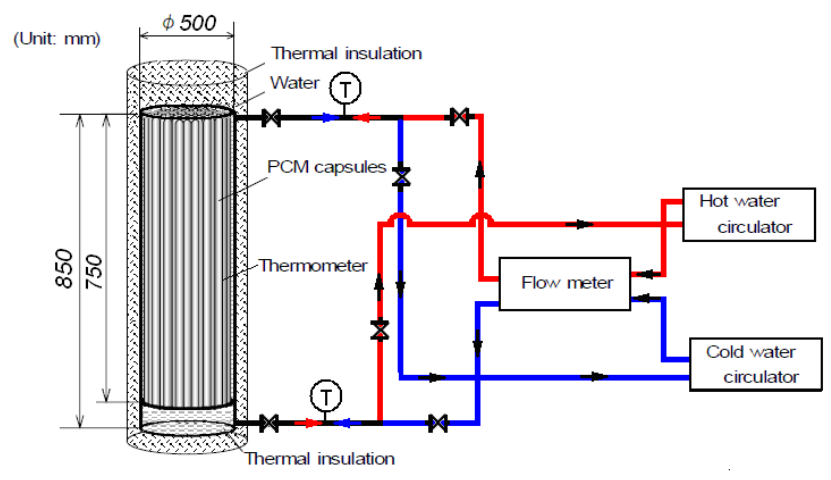

(b)

Fig. 1 Experimental setup (a) Photographic view (b) Schematic of LTES system

The schematic diagram of the experimental apparatus is shown in Fig. 1. The stainless-steel LTES tank, insulated with thermal insulation material of $50 \mathrm{~mm}$ in thickness, had a capacity of $166 \mathrm{~L}$ (500 mm diameter and $850 \mathrm{~mm}$ height), in which 27 heat storage tubes with the 76 $\mathrm{mm}$ in inner diameter and $750 \mathrm{~mm}$ in height were uniformly packed and supported by a wire mesh. The paraffin and EG/paraffin composite PCM were used as PCMs and water was used as the HTF. A hot water tank was used for heating during the heat storage and cold water from a cold water tank was used for cooling during the heat retrieval. During heat storage, the hot water was supplied from the top of LTES tank and was drained from the bottom, whereas, during heat retrieval, the flowing direct for the cooling water was just reversed. The temperatures of HTF at the inlet and the outlet were measured by two PT1000 platinum resistance temperature sensors. 
The schematic of the cylindrical heat storage unit, as shown in Fig. 2, was a vertical tube (stainless steel, outer diameter of $78 \mathrm{~mm}$ and wall thickness of $1 \mathrm{~mm}$ ) in which the PCM was impregnated. Four thermocouples (K-type) were used to measure the temperature of the PCM and were fixed near the centre axis of the tube, as shown in Fig. 2 (b). The heat storage unit which was equipped with thermocouples was set at the center of the LTES tank. The temperature variations of PCM during heat storage and retrieval were monitored and collected using a data logger.

Initially, $80 \%$ of the tube volume was filled with the solid PCM at a room temperature of 28 oC. The remaining $20 \%$ of the volume was left to accommodate the volume increase of the PCM during melting. Water was used as HTF, whose temperature at the inlet of the heat storage unit was kept at $85{ }^{\circ} \mathrm{C}$ during heat storage and was kept at $28{ }^{\circ} \mathrm{C}$ during heat retrieval. There were three different flow rates of the hot water $(100,150,200 \mathrm{~L} / \mathrm{h})$ during heat storage and three different flow rates of the cooling water $(150,200,250 \mathrm{~L} / \mathrm{h})$ during heat retrieval.

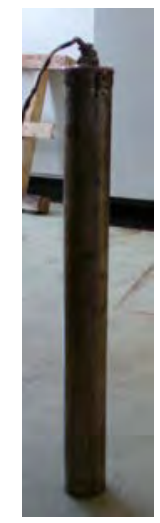

(a)

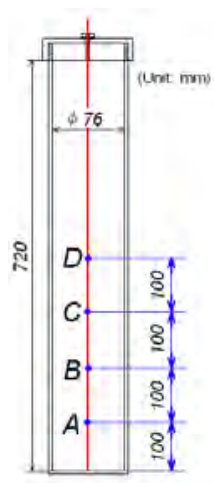

(b)

Fig. 2. LTES unit (a) and locations of the thermocouples (b)

After heat storage of the LTES tank, the heat retrieval experiments were carried out in both continuous and step-by-step heat retrieval modes. In the continuous mode, the cold water of $28{ }^{\circ} \mathrm{C}$ continuously flew through the storage tank until the temperature of the water at the outlet reached $28{ }^{\circ} \mathrm{C}$; while in the step-by-step heat retrieval mode, the cold water at $28{ }^{\circ} \mathrm{C}$ was impregnated in the LTES tank and had been kept there for one hour. Then, the heated HTF was withdrawn and at the same time the temperature was recorded. The above process was repeated until the temperature of the withdrawn HTF is below $35{ }^{\circ} \mathrm{C}$. Nearly five batches of hot water could be withdrawn from the LTES tank in the step-by-step heat retrieval mode.

\section{Results and discussion}

\subsection{Heat storage and retrieval performance}

In the experiment for investigating the heat storage and retrieval performance of the LTES system, flow rate of the water was kept constantly at $150 \mathrm{~L} / \mathrm{h}$ and the inlet HTF temperature during the heat storage and retrieval was $85 \mathrm{oC}$ and $28 \mathrm{oC}$, respectively. The heat retrieval is in the continuous mode.

Figure 3 shows the temperature evolutions at the tested point $C$ (as shown in Fig. 2) of pure paraffin and EG/paraffin composite during heat storage and retrieval circle. It can be found in 
Fig. 3 that the tested point in both pure paraffin and EG/paraffin composite experienced three steps during melting, viz.: the sensible heat storage where the temperature rose rapidly, the latent heat storage (phase change) with the isothermal behavior and the following sensible heat storage where the temperature rose rapidly again until it reached the thermal equilibrium. The similar analysis was also effective during freezing. However, a discrepancy between the measured results of pure paraffin and those of the EG/paraffin composite was observed. The heat storage and retrieval durations of the LTES tank with EG/paraffin composite was much shorter than those with pure paraffin, i.e., the addition of EG drastically enhanced the heat transfer of inner PCM.

It took about $8000 \mathrm{~s}$ for pure paraffin to finish the heat storage, whereas, it took only $4500 \mathrm{~s}$ for EG/paraffin composite to reach the temperature equilibrium with the heating source, showing a $44 \%$ time reduction compared with that for pure paraffin. It was obvious that the heat storage rate of the composite PCM was higher than that of pure paraffin. It can also be seen from Fig. 3 that it took about $18000 \mathrm{~s}$ for the temperature of pure paraffin to drop from $85{ }^{\circ} \mathrm{C}$ to $30{ }^{\circ} \mathrm{C}$, whereas, it took only 5500 s for EG/paraffin composite to complete the heat retrieval, indicating a $69 \%$ reduction in the heat retrieval duration.

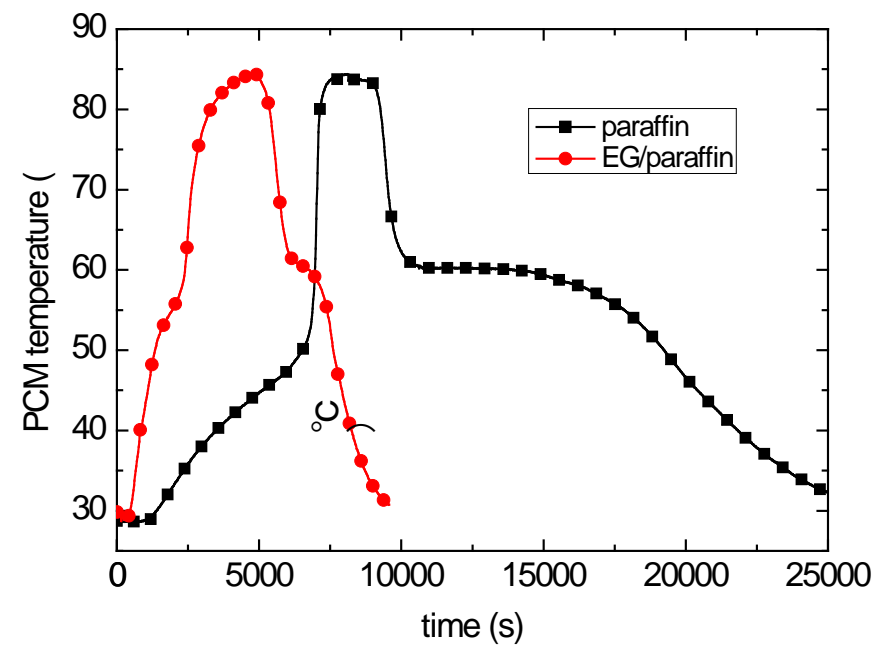

Fig. 3. Temperature evolutions of the LTES unit with pure paraffin and EG/paraffin composite during heat storage and retrieval circle

The heat storage and retrieval durations were both considerably reduced for EG/paraffin composite which was attributed to the addition of EG. However, it can also be seen that the effect of EG was more significant in heat retrieval than in heat storage. These phenomena can be attributed to the melting/freezing characteristic of each PCM: the melting of pure paraffin was accelerated during heat storage (melting) because of the intensive natural convection in the melted paraffin, whereas the natural convection did not play significant role in the heat transfer during heat retrieval (freezing); as for EG/paraffin composite, the natural convection could be neglected during both melting and freezing because of the existence of EG.

The outlet temperature evolutions of HTF during heat storage and retrieval are shown in Fig. 4(a) and (b), respectively. During heat storage, the outlet temperature of the HTF in the LTES system filled with EG/paraffin composite was more rapidly raised to the inlet temperature $\left(85^{\circ} \mathrm{C}\right)$ than in the system filled with paraffin, as shown in Fig. 4(a),. Moreover, in the earlier stage of the heat storage the outlet temperature of the HTF for the LTES system filled with EG/paraffin composite was higher than that with paraffin. These phenomena both indicated 
the system filled with EG/paraffin composite had a better heat transfer performance than the system with paraffin.

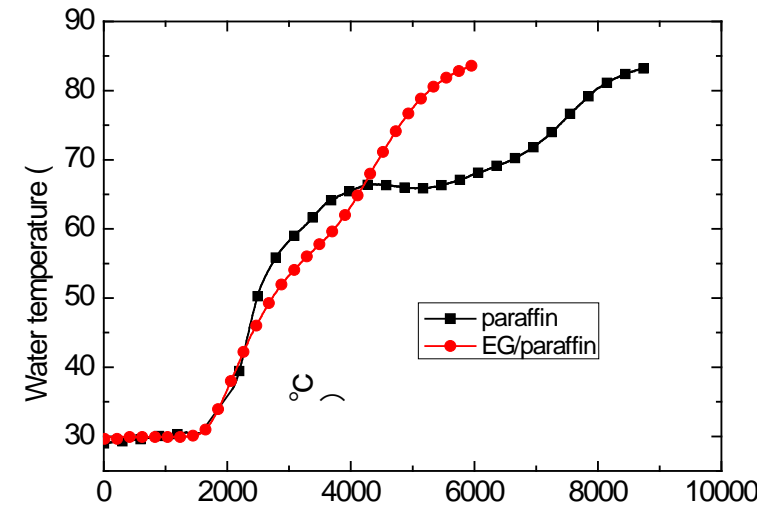

(a)

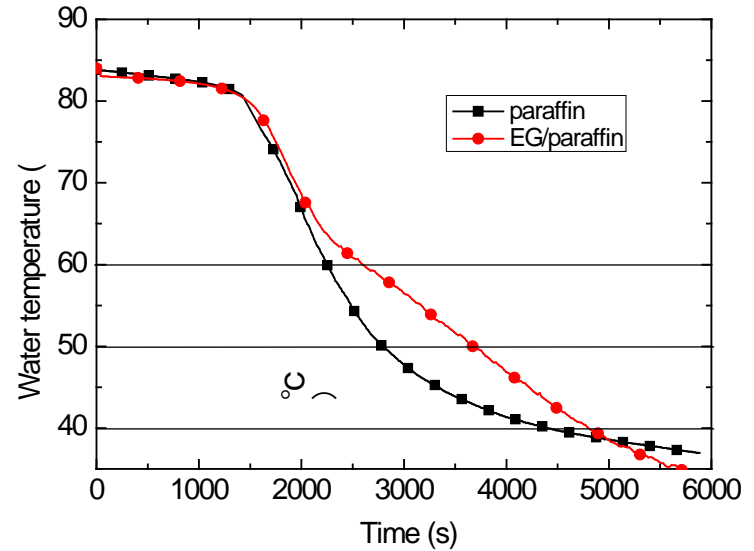

(b)

Fig. 4. Outlet temperature evolutions of the HTF during (a) heat storage and (b) heat retrieval

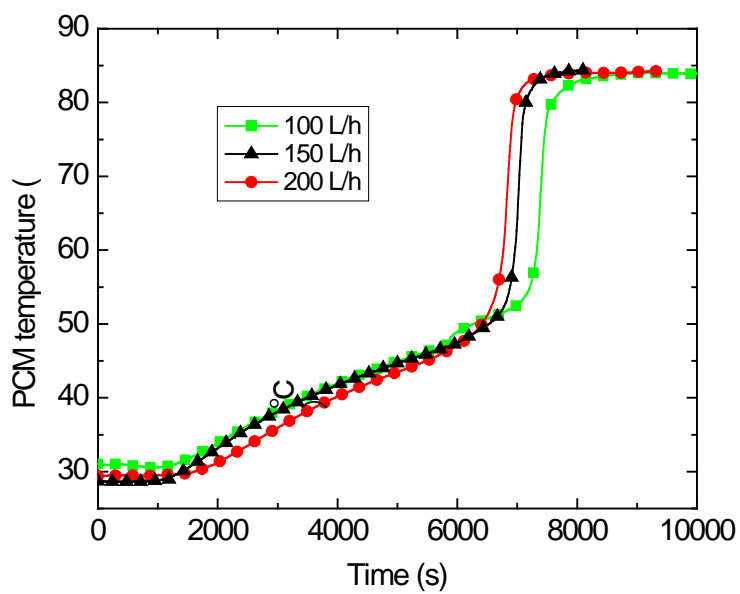

(a)

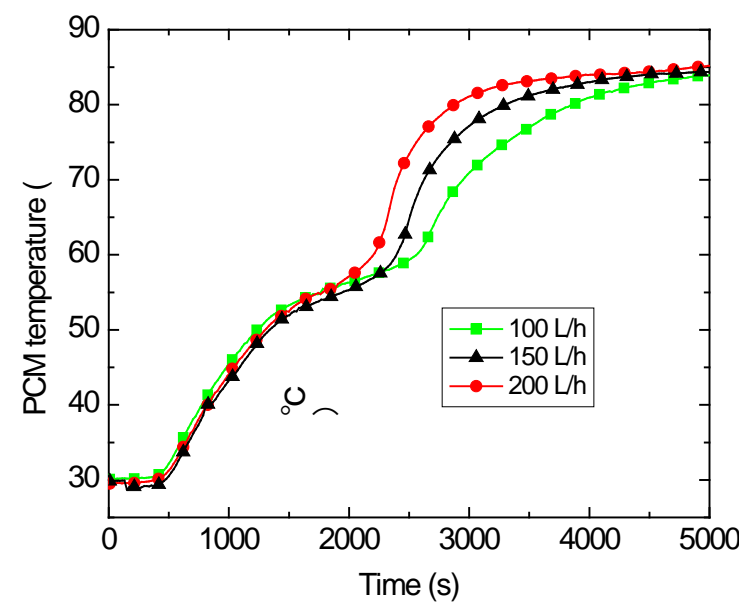

(b)

Fig. 5. Temperature evolutions of the pure paraffin (a) and EG/paraffin composite (b) with the varying flow rate of the HTF during heat storage

As well known, for a LTES system, it is important to have a large heat storage capacity; however, the most important performance is whether it can supply a high heat retrieval power. In an excellent LTES system, the HTF should be heated up rapidly and the temperature of the HTF can be raised to a higher value so as to meet the requirement of the user as the HTF flows through it during heat retrieval. As can be seen from Fig. 4 (b) for the LTES system filled with paraffin/EG composite, the outlet temperature of the HTF could maintain a high level in a longer term than that with paraffin, such as the outlet temperature of HTF of the LTES system filled with paraffin/EG composite could be maintained above $50{ }^{\circ} \mathrm{C}$ for another more $1000 \mathrm{~s}$ than that with paraffin. Thus, it is indicated the stored thermal energy can be rapidly and intensively released in the system filled with paraffin/EG composite, which was significant for the utilization of the LTES system. 


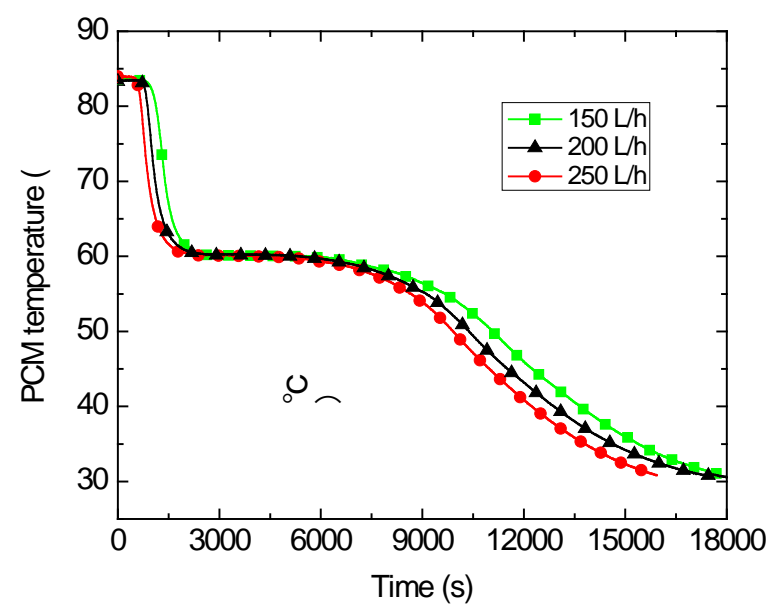

(a)

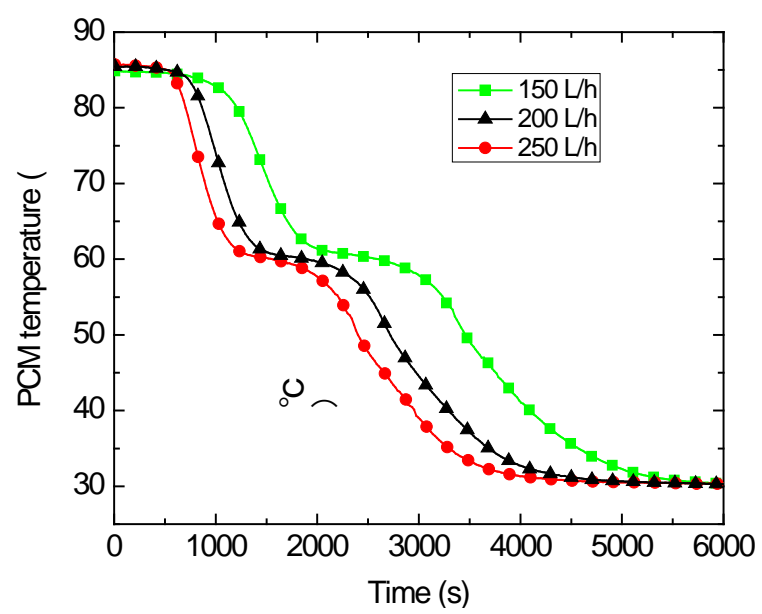

(b)

Fig. 6. Temperature evolutions of the pure paraffin (a) and EG/paraffin composite (b) with the varying flow rate of the HTF during heat retrieval

\subsection{Influence of the flow rate of the HTF on the heat storage and retrieval performance}

Figure 5 shows the temperature evolutions of PCM when varying flow rate of the HTF during heat storage, where Fig. 5(a) is temperature evolutions of the paraffin and Fig. 5(b) is temperature evolutions of the paraffin/EG composite. Figure 6 shows the temperature evolutions of PCM when varying flow rate of the HTF during heat retrieval.

From Fig. 5 and 6, it can be obviously seen that a higher flow rate of the HTF led to a better heat transfer performance and consequently a more rapid heat storage and retrieval. To increase the flow rate is always an effective and positive means during heat storage, whereas higher flow rate of the HTF may cause lower outlet temperature of the HTF during heat retrieval though it can enhance the heat retrieval power.

\subsection{Test for the step-by-step heat retrieval mode}

For the utilizations of the LTES system, the heat retrieval mode is not only continuous but also discontinuous, for example, the requirement of the hot water is intermittent in the domestic hot water system. Thus, the information about the step-by-step heat retrieval mode of the LTES system was also necessary and the retrieval performance of the LTES system was investigated in such case. Figure 7(a) shows the temperature evolutions of PCM and Fig. 7(b) shows the temperature evolutions of the outlet HTF during step-by-step heat retrieval. In each figure, the performance of the LTES system with paraffin was compared with that with EG/paraffin composite. The experimental result indicated: 1. There is a large difference between the temperature evolutions of the pure paraffin and EG/paraffin composite. This is because the EG/paraffin composite with high thermal conductivity is more sensitive to the varying of the HTF temperature and can quickly response this varying; 2 . The temperature evolutions of the outlet HTF in the two LTES systems are almost the same with each other. This is because the waiting duration of one hour in each step is an enough time period to allow new temperature equilibrium is reached and maintained between PCM and HTF for both two LTES systems. 


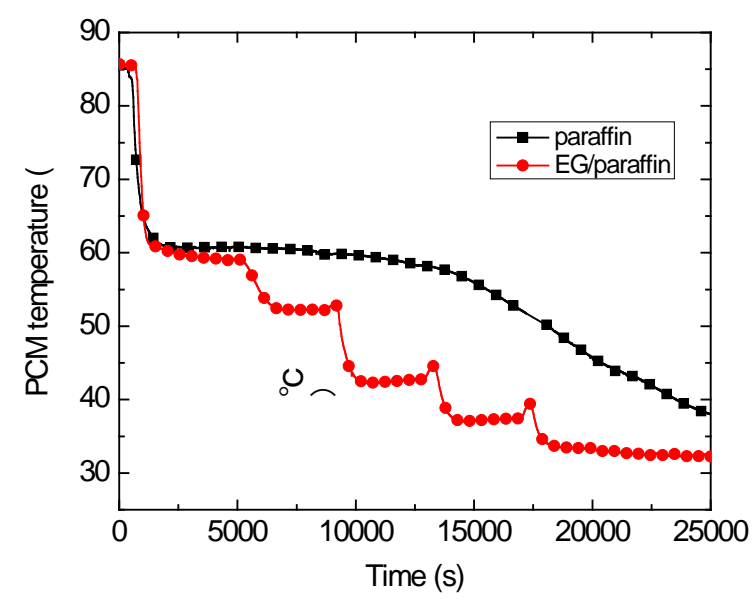

(a)

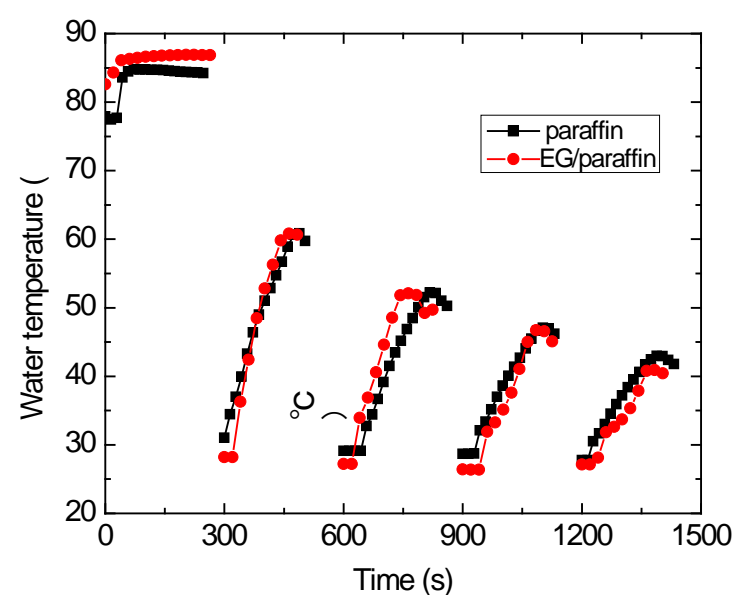

(b)

Fig. 7. Temperature evolutions of PCM (a) and the outlet HTF (b) during step-by-step heat retrieval

\section{Conclusions}

Paraffin/EG composite PCM with 7\% mass fraction of EG was prepared for enhancing the heat transfer of paraffin. The paraffin/EG composite PCM and the paraffin were used in a shell and tube heat storage system and the performance of the LTES system was experimentally investigated. The following conclusions were drawn:

1. The utilization of paraffin/EG composite PCM greatly enhanced the heat storage/retrieval rates of the LTES system. The LTES system with paraffin/EG composite PCM, under the operation condition (flow rates: $150 \mathrm{~L} / \mathrm{h}$ during both heat storage and heat retrieval; the inlet temperature of HTF: $28{ }^{\circ} \mathrm{C}$ during heat retrieval and $85{ }^{\circ} \mathrm{C}$ during heat storage), showed a $44 \%$ reduction in heat storage duration and a nearly $69 \%$ reduction in the retrieval duration, respectively, compared to those for pure paraffin.

2. The most outstanding advantage, for the LTES system filled with paraffin/EG composite, was that the outlet temperature of HTF can be maintained at a higher level in a longer term than that with paraffin, which was significant for the utilization of the LTES system.

3. A higher flow rate of the HTF led to a better heat transfer performance and consequently more rapid heat storage and retrieval. It is positive for heat storage, whereas higher flow rate of the HTF may cause lower outlet temperature of the HTF during heat retrieval though it can enhance the heat retrieval power.

4. There was a large difference between the temperature evolutions of the pure paraffin and paraffin/EG composite PCM in the step-by-step heat retrieval mode, whereas the temperature evolutions of the outlet HTF in the two LTES systems were almost the same with each other.

\section{References}

[1] G.A. Lane, Solar heat storage: latent heat materials, background and scientific principles vol. I. Florida: CRC Press Inc, 1983. 
[2] A. Felix Regin, S.C. Solanki, J.S. Saini, Heat transfer characteristics of thermal energy storage system using PCM capsules: A review, Renewable and Sustainable Energy Reviews12, 2008, pp. 2438-2458.

[3] C. Arkar, B. Vidrihb, S. Medveda, Efficiency of free cooling using latent heat storage integrated into the ventilation system of a low energy building, International Journal of Refrigeration 30, 2007, pp. 134-143.

[4] A. Benmansour, M.A. Hamdan, A. Bengeuddach, Experimental and numerical investigation of solid particles thermal energy storage unit, Applied Thermal Energy 26, 2006, pp. 513-518.

[5] F. Agyenim, N. Hewitt, P. Eames, M. Smyth, A review of materials, heat transfer and phase change problem formulation for latent heat thermal energy storage systems (LHTESS), Renewable and Sustainable Energy Reviews 14, 2010, pp. 615-628.

[6] A. Trp, An experimental and numerical investigation of heat transfer during technical grade paraffin melting and solidification in a shell-and-tube latent thermal energy storage unit, Solar Energy 79, 2005, pp. 648-660.

[7] H.A. Adine, H.E. Qarnia, Numerical analysis of the thermal behaviour of a shell-and-tube heat storage unit using phase change materials, Applied Mathematical Modelling 33, 2009, pp. 2132-2144

[8] F. Agyenim, P. Eames, M. Smyth, A comparison of heat transfer enhancement in a medium temperature thermal energy storage heat exchanger using fins, Solar Energy 83, 2009, pp. 1509-1520.

[9] M. Esen, A. Durmus, Geometric design of solar-aided latent heat store depending on various parameters and phase change materials. Solar Energy 62, 1998, pp. 19-28.

[10]F. Agyenim, P. Eames, M. Smyth, Heat transfer enhancement in medium temperature thermal energy storage system using a multitube heat transfer array. Renewable Energy 35, 2010, pp. 198-207.

[11]A.A. Ghoneim, Comparison of theoretical models of phase-change and sensible heat storage for air and water-based solar heating systems, Solar Energy 42, 1989, pp. 209220.

[12]L. Xia, P. Zhang, R.Z. Wang, Preparation and thermal characterization of expanded graphite/paraffin composite phase change material, Carbon 48, 2010, pp. 2538-2548 